\title{
On the solutions of the generalized Lotka-Volterra system
}

\author{
Lazhar Bougoffa ${ }^{1, *}$ and Ammar Khanfer ${ }^{2, * *}$ \\ ${ }^{1}$ Al Imam Mohammad Ibn Saud Islamic University, Faculty of Science, Department of Mathematics, \\ P.O. Box 90950, Riyadh 11623, Saudi Arabia. \\ ${ }^{2}$ Al Imam Mohammad Ibn Saud Islamic University, Faculty of Science, Department of Mathematics, \\ P.O. Box 90950, Riyadh 11623, Saudi Arabia.
}

\begin{abstract}
In this article, under suitable conditions on the coefficients, we derive closed-form solutions and implicit solutions of the generalized Lotka-Volterra system.
\end{abstract}

\section{Introduction}

The Lotka-Volterra equations [1-9], also known as the predatorprey model, are a coupled system of first-order non-linear differential equations frequently used to describe the dynamics of biological systems in which two species interact, one as a predator and the other as prey. The populations change through time according to the following pair of equations:

$$
\left\{\begin{array}{l}
\frac{d x}{d t}=\alpha x-\beta x y, \\
\frac{d y}{d t}=-\gamma y+\delta x y,
\end{array}\right.
$$

where $x$ is the number of prey (for example, rabbits); $y$ is the number of some predator (for example, foxes); $\frac{d y}{d t}$ and $\frac{d x}{d t}$ represent the growth rates of the two populations over time $t$, and $\alpha, \beta, \gamma, \delta$ are positive real parameters and interpreted as follows: $\alpha$ represents the birth rate of the prey, $\beta$ represents the death rate of prey due to attacks from predators, $\gamma$ represents the natural death of predators in the absence of prey and $\delta$ represents the birth rate of the predator, which measures the efficiency and propagation rate of predators in the presence of prey.

The model has been proposed independently by Lotka [1, 2] and Volterra [3, 4]. A derivation of this system can be found in the original paper by Volterra [2] and [3]-[9].

This system has played a fundamental role in the development of mathematical ecology and population dynamics. This stems from the fact that it has been one of the oldest models for a general pattern in nature, thus providing a foundation for more sophisticated and restrictive models. Explicit solutions to this system can be found by eliminating time from the two differential equations above to get

$$
\frac{d y}{d x}=-\frac{y}{x} \frac{\delta x-\gamma y}{\beta y-\alpha},
$$

which is a separable ODE, and the explicit solution of Eq. (2) can be obtained as

$$
\beta y-\alpha \ln y+\delta x-\gamma \ln x=C,
$$

\footnotetext{
*e-mail: lbbougoffa@imamu.edu.sa

**e-mail: ahkhanfer@imamu.edu.sa
} 
where $C$ is a constant of integration.

Some researchers proposed controlled models where a control is added to both equations, so they become:

$$
\left\{\begin{aligned}
\frac{d x}{d t} & =\alpha x-\beta x y-x u_{\text {prey }}(t), \\
\frac{d y}{d t} & =-\gamma y+\delta x y-y u_{\text {pred }}(t),
\end{aligned}\right.
$$

where $u_{\text {pred }}(t)$ is the control variable for the prey population, acting on the predator dynamics, and similarly for $u_{\text {prey }}(t)$. This is to allow the prey population to achieve a desired state $x_{d}$ for the prey and a desired state $y_{d}$ for the predator.

Recently, Lacitignola et al. [10] both applied the Z-type control method to the classical Lotka-Volterra model and the generalized model respectively, in order to maintain species from extinction and to enhance ecosystem stability. Prey and predator populations have been proved to converge to the desired state exponentially. Two cases have been considered: A direct control is proposed to control the prey and the predator populations simultaneously, and the case of indirect control has been applied to one of the species, but the other one needs to be controlled.

In fact, the direct controlled Lotka-Volterra model can be written as:

$$
\left\{\begin{aligned}
\frac{d x}{d t} & =\left(\alpha-u_{\text {prey }}(t)\right) x-\beta x y \\
\frac{d y}{d t} & =-\left(\gamma+u_{\text {pred }}(t)\right) y+\delta x y
\end{aligned}\right.
$$

where $\alpha-u_{\text {prey }}(t)$ and $\gamma+u_{\text {pred }}(t)$ are functions of $t$.

This has motivated us to investigate the case where all $\alpha(t), \beta(t), \gamma(t)$ and $\delta(t)$ are differentiable functions of $t$.

The purpose of this paper is to present, under suitable conditions on the coefficients, new exact solutions of the system:

$$
\left\{\begin{aligned}
\frac{d x}{d t} & =\alpha(t) x-\beta(t) x y \\
\frac{d y}{d t} & =-\gamma(t) y+\delta(t) x y
\end{aligned}\right.
$$

It is worth noting that Volterra has made the following assumptions:

1. Presence of the predator depends on the presence of the prey.

2. Prey population grows up exponentially without bounds in the absence of predators.

3. Prey can always find food.

4. Both species are uniformly distributed in their habitat.

Under the aforementioned assumptions, we can propose the following compatibility condition as a natural law for this specific habitat:

If $\alpha$ declines then $\gamma$ increases due to starvation, which in turn causes $\alpha$ to increase. On the other hand, if $\alpha$ increases then $\gamma$ declines due to availability of food, which in turn causes $\alpha$ to decline due to the abundance of attacks from the predators.

This condition seems natural and satisfies the assumptions, and most importantly leads to the following population cycle:

$$
\alpha \uparrow \Rightarrow \gamma \downarrow \Rightarrow \beta \uparrow \Rightarrow \alpha \downarrow \Rightarrow \gamma \uparrow \Rightarrow \beta \downarrow \Rightarrow \alpha \uparrow .
$$




\section{The Exact Solutions}

\subsection{The First Exact Solutions}

The first step is to change system (6) into an equivalent system. For this, We perform a change of dependent variables as follows:

$$
x=e^{u} \text { and } y=e^{v} .
$$

Substituting (8) into (6), we obtain

$$
\left\{\begin{array}{l}
\frac{d u}{d t}=\alpha(t)-\beta(t) e^{v} \\
\frac{d v}{d t}=-\gamma(t)+\delta(t) e^{u}
\end{array}\right.
$$

Thus

$$
\left\{\begin{aligned}
\frac{d u}{d t}+\beta(t) e^{v} & =\alpha(t), \\
-\frac{d v}{d t}+\delta(t) e^{u} & =\gamma(t) .
\end{aligned}\right.
$$

Rewrite system (10) as

$$
\left\{\begin{aligned}
\frac{1}{\alpha(t)} \frac{d u}{d t}+\frac{\beta(t)}{\alpha(t)} e^{v} & =1, \\
-\frac{1}{\gamma(t)} \frac{d v}{d t}+\frac{\delta(t)}{\gamma(t)} e^{u} & =1,
\end{aligned}\right.
$$

or

$$
\left\{\begin{aligned}
\frac{1}{\alpha(t)} \frac{d u}{d t}+e^{\ln \left(\frac{\beta(t)}{\alpha(t)}\right)+v} & =1, \\
-\frac{1}{\gamma(t)} \frac{d v}{d t}+e^{\ln \left(\frac{\delta(t)}{\gamma(t)}\right)+u} & =1 .
\end{aligned}\right.
$$

Now by the change of variables

$$
U=\ln \left(\frac{\delta(t)}{\gamma(t)}\right)+u \text { and } V=\ln \left(\frac{\beta(t)}{\alpha(t)}\right)+v
$$

Substituting (13) into (12), we obtain

$$
\left\{\begin{aligned}
\frac{1}{\alpha(t)} \frac{d U}{d t}-\frac{1}{\alpha(t)} \frac{d}{d t}\left(\ln \frac{\delta(t)}{\gamma(t)}\right)+e^{V} & =1, \\
-\frac{1}{\gamma(t)} \frac{d V}{d t}+\frac{1}{\gamma(t)} \frac{d}{d t}\left(\ln \frac{\beta(t)}{\alpha(t)}\right)+e^{U}= & 1 .
\end{aligned}\right.
$$

If we assume that

$$
\frac{1}{\alpha(t)} \frac{d}{d t}\left(\ln \frac{\delta(t)}{\gamma(t)}\right)=K_{1}
$$

and

$$
\frac{1}{\gamma(t)} \frac{d}{d t}\left(\ln \frac{\beta(t)}{\alpha(t)}\right)=K_{2},
$$

where $K_{1}$ and $K_{2}$ are two constants, then system (14) reduces to

$$
\left\{\begin{aligned}
\frac{1}{\alpha(t)} \frac{d U}{d t}+e^{V} & =1+K_{1}, \\
-\frac{1}{\gamma(t)} \frac{d V}{d t}+e^{U} & =1-K_{2} .
\end{aligned}\right.
$$


Clearly, $(U, V)=\left(\ln \left(1-K_{2}\right), \ln \left(1+K_{1}\right)\right), K_{1}>-1, K_{2}<1$ is a solution to system (17). We then get from (13)

$$
u=\ln \left(1-K_{2}\right)-\ln \left(\frac{\delta(t)}{\gamma(t)}\right) \text { and } v=\ln \left(1+K_{1}\right)-\ln \left(\frac{\beta(t)}{\alpha(t)}\right) .
$$

Consequently,

$$
x=\left(1-K_{2}\right) \frac{\gamma(t)}{\delta(t)} \text { and } y=\left(1+K_{1}\right) \frac{\alpha(t)}{\beta(t)} .
$$

Thus we have proved

Theorem 1 Let $\alpha(t), \beta(t), \gamma(t)$ and $\delta(t)$ be positive and differentiable functions. If we assume that

$$
\frac{\delta^{\prime}(t)}{\delta(t)}-\frac{\gamma^{\prime}(t)}{\gamma(t)}=K_{1} \alpha(t)
$$

and

$$
\frac{\beta^{\prime}(t)}{\beta(t)}-\frac{\alpha^{\prime}(t)}{\alpha(t)}=K_{2} \gamma(t)
$$

then the exact solution to the general nonlinear system (6) is given by (19).

The condition (20) implies that: If $\gamma \uparrow$ then $\beta \downarrow$ and /or $\alpha \uparrow$, and If $\gamma \downarrow$ then $\beta \uparrow$ and /or $\alpha \downarrow$. The condition (21) implies: If $\alpha \uparrow$ then $\gamma \downarrow$ and /or $\delta \uparrow$, and If $\alpha \downarrow$ then $\gamma \uparrow$ and /or $\delta \downarrow$.

The two conditions are consistent with the compatibility condition and the population cycle. Now we consider the simplest case of Theorem 1, namely the case when $\alpha(t)=\alpha, \beta(t)=$ $\beta, \gamma(t)=\gamma$ and $\delta(t)=\delta$. Thus the conditions of this theorem are satisfied, and we find $K_{1}=K_{2}=0$. Hence Let $\alpha, \beta, \gamma$ and $\delta$ be positive constants, then the exact solution to the general nonlinear system (6) is given as

$$
x=\frac{\gamma}{\delta} \text { and } y=\frac{\alpha}{\beta} .
$$

This solution represents a fixed point (population equilibrium). This means that levels of population at which this equilibrium is achieved depend on the chosen values of the parameters $\alpha, \beta, \gamma$ and $\delta$.

\subsection{The second Exact Solutions}

Differentiating both sides of the second equation of (10), we obtain

$$
v^{\prime \prime}=-\gamma^{\prime}(t)+\delta^{\prime}(t) e^{u}+\delta(t) u^{\prime} e^{u} .
$$

Eliminating $u^{\prime}$ and $e^{u}$ from (23) by using (10), we obtain a second order differential equation in $v$ :

$$
v^{\prime \prime}=\left(\frac{\delta^{\prime}(t)}{\delta(t)}+\alpha(t)\right) v^{\prime}-\beta(t) v^{\prime} e^{v}-\beta(t) \gamma(t) e^{v}+\alpha(t) \gamma(t)-\gamma^{\prime}(t)+\gamma(t) \frac{\delta^{\prime}(t)}{\delta(t)} .
$$

Multiplying both sides of Eq.(24) by $e^{-\int f(t) d t}$, where $f(t)=\frac{\delta^{\prime}(t)}{\delta(t)}+\alpha(t)$, to obtain

$$
\left(e^{-\int f(t) d t} v^{\prime}\right)^{\prime}=-\beta(t) e^{-\int f(t) d t}\left(v^{\prime}+\gamma(t)\right) e^{v}+e^{-\int f(t) d t} g(t),
$$

where $g(t)=\alpha(t) \gamma(t)-\gamma^{\prime}(t)+\gamma(t) \frac{\delta^{\prime}(t)}{\delta(t)}$.

Multiplying now both sides of Eq.(25) by $e^{\int \gamma(t) d t}$, we obtain

$$
e^{\int \gamma(t) d t}\left(e^{-\int f(t) d t} v^{\prime}\right)^{\prime}=-\beta(t) e^{-\int f(t) d t}\left(v^{\prime}+\gamma(t)\right) e^{v+\int \gamma(t) d t}+e^{-\int(f(t)+\gamma(t)) d t} g(t) .
$$


So that

$$
\left(e^{-\int f(t) d t} v^{\prime}\right)^{\prime}=-\beta(t) e^{-\int(f(t)+\gamma(t)) d t}\left(e^{v+\int \gamma(t) d t}\right)^{\prime}+e^{-\int f(t) d t} g(t) .
$$

If we assume that there is a constant $K$ such that

$$
\beta(t) e^{-\int(f(t)+\gamma(t)) d t}=K
$$

Then, Eq.(27) becomes

$$
\left(e^{-\int f(t) d t} v^{\prime}\right)^{\prime}=-K\left(e^{v+\int \gamma(t) d t}\right)^{\prime}+e^{-\int f(t) d t} g(t) .
$$

Hence,

$$
e^{-\int f(t) d t} v^{\prime}=-K e^{\int \gamma(t) d t} e^{v}+\int e^{-\int f(t) d t} g(t) d t+C,
$$

where $C$ is a constant of integration.

Substituting the second equation of the system (10) into the LHS of (30), we obtain

$$
e^{-\int f(t) d t}\left(-\gamma(t)+\delta(t) e^{u}\right)=-K e^{\int \gamma(t) d t} e^{v}+\int e^{-\int f(t) d t} g(t) d t+C
$$

Hence,

$$
\delta(t) e^{u}+K e^{\int(f(t)+\gamma(t)) d t} e^{v}=\gamma(t)+e^{\int f(t) d t} \int e^{-\int f(t) d t} g(t) d t+C e^{\int f(t) d t} .
$$

Consequently,

$$
\delta(t) x+K e^{\int(f(t)+\gamma(t)) d t} y=\gamma(t)+e^{\int f(t) d t}\left(\int e^{-\int f(t) d t} g(t) d t+C\right) .
$$

Using the above condition (28), thus (33) becomes

$$
\delta(t) x+\beta(t) y=\gamma(t)+e^{\int f(t) d t}\left(\int e^{-\int f(t) d t} g(t) d t+C\right) .
$$

The substitution of (34) into the original system (6) leads to the two Bernoulli's equations:

$$
\frac{d x}{d t}=\phi(t) x+\delta(t) x^{2}
$$

and

$$
\frac{d y}{d t}=\psi(t) y-\beta(t) y^{2}
$$

where

$$
\phi(t)=\alpha(t)-\gamma(t)-e^{\int f(t) d t}\left(\int e^{-\int f(t) d t} g(t) d t+C\right)
$$

and

$$
\psi(t)=e^{\int f(t) d t}\left(\int e^{-\int f(t) d t} g(t) d t+C\right)
$$

Then the general solutions of (35) and (36) are given, respectively, by

$$
x=\left[-e^{-\int \phi(t) d t} \int e^{\int \phi(t) d t} \delta(t) d t+C_{1} e^{-\int \phi(t) d t}\right]^{-\frac{1}{2}}
$$

and

$$
y=\left[e^{-\int \psi(t) d t} \int e^{\int \psi(t) d t} \beta(t) d t+C_{2} e^{-\int \psi(t) d t}\right]^{-\frac{1}{2}},
$$

respectively, where $C_{1}$ and $C_{2}$ are two constants.

Thus 
Lemma 2 Let $\alpha(t), \beta(t), \gamma(t)$ and $\delta(t)$ be positive and differentiable functions. If there exists a constant such that $(28)$ is satisfied, then the exact solution $(x, y)$ to the general nonlinear system (6) is given by (39) and (40).

\section{The Implicit Solutions}

Furthermore, if we assume that $\alpha(t)=\alpha$ and $\gamma(t)=\gamma$. Then by eliminating time from the two differential equations of system (17) to get

$$
-\frac{\gamma}{\alpha} \frac{d U}{d V}=\frac{1+K_{1}-e^{V}}{1-K_{2}-e^{U}}
$$

which gives

$$
\gamma\left(1-K_{2}\right) U-\gamma e^{U}=-\alpha\left(1+K_{1}\right) V+\alpha e^{V}+C_{1},
$$

where $C_{1}$ is a constant of integration. It follows that

$$
\begin{gathered}
\alpha\left(1+K_{1}\right) \ln \beta(t)+\gamma\left(1-K_{2}\right) \ln \delta(t)-\alpha\left(1+K_{1}\right) \ln \alpha-\gamma\left(1-K_{2}\right) \ln \gamma \\
+\alpha\left(1+K_{1}\right) v+\gamma\left(1-K_{2}\right) u-\beta(t) e^{v}-\delta(t) e^{u}=C_{1} .
\end{gathered}
$$

Hence, the implicit form of the solution can be obtained as

$$
\beta(t) y+\delta(t) x-\alpha\left(1+K_{1}\right) \ln y-\gamma\left(1-K_{2}\right) \ln x=\varphi(t),
$$

where

$$
\varphi(t)=\alpha\left(1+K_{1}\right) \ln \beta(t)-\gamma\left(1-K_{2}\right) \ln \delta(t)-\alpha\left(1+K_{1}\right) \ln \alpha+\gamma\left(1-K_{2}\right) \ln \gamma-C_{1} .
$$

Thus we have proved

Lemma 3 Let $\alpha(t)=\alpha>0, \gamma(t)=\gamma>0$ be two constants and $\beta(t), \delta(t)$ be two positive and differentiable functions. Under the same hypothesis of Lemma 1. Then the general solution to the nonlinear system (6) is given by the implicit form (44) and (45).

\section{References}

[1] A.J. Lotka, Contribution to the Theory of Periodic Reaction, J. Phys. Chem., 14 (3), pp 271-274, (1910)

[2] V. Volterra, Leçon sur la thérie mathématique de la lutte pour la vie, Cahiers Scientifiques, Paris, (1931)

[3] V. Volterra, Variazioni e fluttuazioni del numero dindividui in specie animali conviventi, Mem. Acad. Lincei Roma, 2, 31-113, (1926)

[4] V. Volterra,Variations and fluctuations of the number of individuals in animal species living together in Animal Ecology, Chapman, R.N. (ed), McGrawHill, (1931)

[5] H.I. Freedman, Deterministic Mathematical Models in Population Ecology, Marcel Dekker, (1980)

[6] N.S. Goel, S.C. Maitra, E.W. Montroll, On the Volterra and Other Non-Linear Models of Interacting Populations, Rev. Mod. Phys., 43, 231, (1971)

[7] H.I. Freedman, Deterministic Mathematical Models in Population Ecology, Marcel Dekker, (1980)

[8] A.A. Berryman, The Origins and Evolution of Predator-Prey Theory, Ecology, 73(5), 1530-1535, (1992)

[9] F. Brauer and C. Castillo-Chavez, Mathematical Models in Population Biology and Epidemiology, Springer-Verlag, (2000)

[10] D. Lacitignola, F. Diele, C. Marangi, A. Provenzale, On the dynamics of a generalized predatorprey system with Z-type control, Mathematical Biosciences, 280, 10-23, (2016) 